\title{
Experimental Composite Tissue Transplantation Models
}

\author{
Maria Siemionow and Serdar Nasir
}

\section{Summary}

Advanced microsurgical techniques have allowed performing different composite tissue allotransplantations (CTA), although immunology and pharmacotherapy still have many unresolved questions. Different CTA models were developed to explore and answer these questions during the past 25 years. Most experimental transplants to date have been performed in rats, and the immune system of the rats has fundamental differences from that of the humans, such as the lack of expression of class II antigens on the endothelial cells. ${ }^{7}$ Over the past 20 years, we have developed 17 CTA models of the rat in our laboratory in an effort to characterize differences in the mechanism of graft acceptance and rejection. These CTA models were tested to (1) determine the mechanism of CTA acceptance and rejection; (2) determine the immunologic pattern of responses of different tissue types in CTA; (3) evaluate the immunologic response of transplant grafts of different dimensions and skin components of the most antigenic of CTA tissues ${ }^{10}$; (4) test the effects of vascularized bone marrow transplantation (VBMT) on immunotolerance; (5) develop new immunosuppressive and immunomodulatory treatment protocols; and (6) correlate histological, morphological, and functional responses of CTA with different immunosuppression protocols. We overwrite CTA models according to the following classification: vascularized bone marrow transplant models, vascularized skin allograft models, composite allograft transplantation models, and other models (Tables 51.1 and 51.2).

\section{Abbreviations}

$\begin{array}{ll}\text { CsA } & \text { Cyclosporine-A } \\ \text { CTA } & \text { Composite tissue allotransplantation } \\ \text { SVC } & \text { Superior vena cava } \\ \text { VBMT } & \text { Vascularized bone marrow } \\ & \text { transplantation }\end{array}$

\section{Vascularized Bone Marrow} Models

Vascularized bone marrow transplantation may be a component of composite tissue allograft (CTA) transplants; and there is evidence that bone marrow cells are tolerogenic and may induce chimerism. Over the years, different models of VBMTs have been introduced and are outlined below.

\section{Hind-Limb Transplant Model}

Limb transplantation was the first VBMT model for this purpose. Black et al. ${ }^{5}$ achieved the first 
Table 51.1. Classification of experimental CTA models.

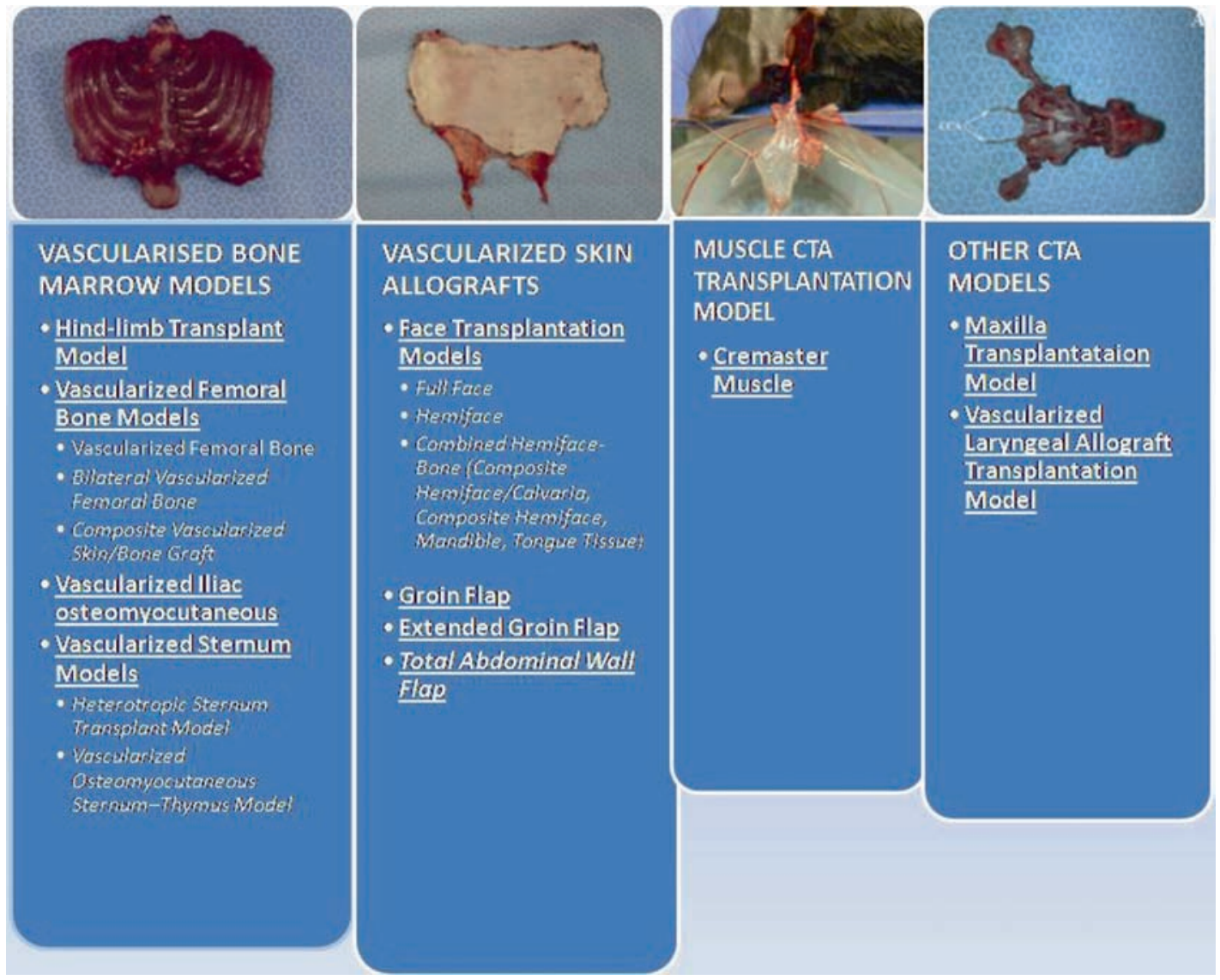

successful semiallogenic limb transplantation in rats in 1982 under the protocol of long-term immunosuppression with cyclosporine-A (CsA) immunotherapy. Zhang et al. ${ }^{25}$ developed an experimental limb transplantation model in mice in 1999. We were the first to report tolerance induction in limb transplants across MHC. ${ }^{15}$

Surgical technique: A limb tissue allograft consisting of distal femur, knee joint, tibia-fibula, and surrounding muscle and skin is harvested using a similar technique in both small ${ }^{18}$ and large animals. ${ }^{11}$ In the donor rat, a circumferential skin incision is made in the proximal one third of the femur. The femoral artery and vein are dissected, clamped, and transected proximal to the superficial epigastric artery. The femoral nerve is dissected and transected $1 \mathrm{~cm}$ distal to the inguinal ligament. The biceps femoris muscle is transected to expose the sciatic nerve. The nerve is then transected proximal to its bifurcation. The limb is amputated at the mid-femoral level. The recipient is pre- pared using a similar technique. The limb is amputated at the mid-femoral level. The donor limb is attached to the recipient limb by a 20 -gauge intramedullary pin and a simple cerclage wire. All large muscle groups are sutured in juxtaposition. Vessels are anastomosed using end-to-end microsurgical anastomosis while a conventional epineural technique is performed for nerve coaptation (Figure 51.1).

Advantages: The limb grafts are vascularized carriers of donor bone marrow and proved to be an important model for elucidating the mechanisms of transplantation tolerance. Limb transplants are composed of many key tissues such as skin, subcutaneous tissue, neuromuscular tissue, and mesenchymal tissues such as bone, bone marrow, muscle, fascia, and cartilage, and the same tissues are a part of many clinical CTAs, including hand or face, larynx, knee, abdominal wall, and so forth.

Disadvantages: Hind-limb transplantation is technically complex and leaves the recipient with 
Table 51.2. Summary of experimental CTA models.

\begin{tabular}{|c|c|c|}
\hline Experimental models & Transplant pedicle & Recipient vessels \\
\hline Vascularized femoral bone & Femoral artery and vein & $\begin{array}{l}\text { Femoral artery and vein or abdominal aorta } \\
\text { and vena cava inferior }\end{array}$ \\
\hline Bilateral vascularized femoral bone & Abdominal aorta and vena cava inferior & Abdominal aorta and vena cava inferior \\
\hline Composite vascularized skin/bone & Femoral artery and vein & Femoral artery and vein \\
\hline Vascularized iliac osteomyocutaneous & Abdominal aorta and iliolumbar vein & Femoral artery and vein \\
\hline Heterotropic sternum & $\begin{array}{l}\text { Bilateral common carotid artery } \\
\text { and unilateral superior vena cava }\end{array}$ & Abdominal aorta and vena cava inferior \\
\hline $\begin{array}{l}\text { Vascularized osteomyocutaneous } \\
\text { sternum-thymus }\end{array}$ & $\begin{array}{l}\text { Unilateral common carotid artery } \\
\text { and external jugular vein }\end{array}$ & Femoral artery and vein \\
\hline Full face & $\begin{array}{l}\text { Bilateral common carotid artery } \\
\text { and bilateral external jugular vein }\end{array}$ & $\begin{array}{l}\text { Bilateral common carotid artery } \\
\text { and bilateral external jugular vein }\end{array}$ \\
\hline Hemiface & $\begin{array}{l}\text { Unilateral common carotid artery } \\
\text { and unilateral external jugular vein }\end{array}$ & $\begin{array}{l}\text { Unilateral common carotid artery } \\
\text { and unilateral external jugular vein }\end{array}$ \\
\hline Composite hemiface/calvaria & $\begin{array}{l}\text { Unilateral common carotid artery } \\
\text { and unilateral external jugular vein }\end{array}$ & $\begin{array}{l}\text { Unilateral common carotid artery } \\
\text { and unilateral external jugular vein }\end{array}$ \\
\hline $\begin{array}{l}\text { Composite hemiface, mandible, } \\
\text { tongue tissue allograft }\end{array}$ & $\begin{array}{l}\text { Unilateral common carotid artery } \\
\text { and unilateral external jugular vein }\end{array}$ & Femoral artery and vein \\
\hline Vascularized groin & Femoral artery and vein & Femoral artery and vein \\
\hline Extended groin & Femoral artery and vein & Femoral artery and vein \\
\hline Total abdominal wall & Bilateral femoral arteries and veins & Bilateral femoral arteries and veins \\
\hline Cremaster muscle & Bilateral femoral arteries and veins & $\begin{array}{l}\text { Unilateral common carotid artery } \\
\text { and unilateral external jugular vein }\end{array}$ \\
\hline Maxilla & $\begin{array}{l}\text { Unilateral common carotid artery } \\
\text { and unilateral external jugular vein }\end{array}$ & Femoral artery and vein \\
\hline
\end{tabular}
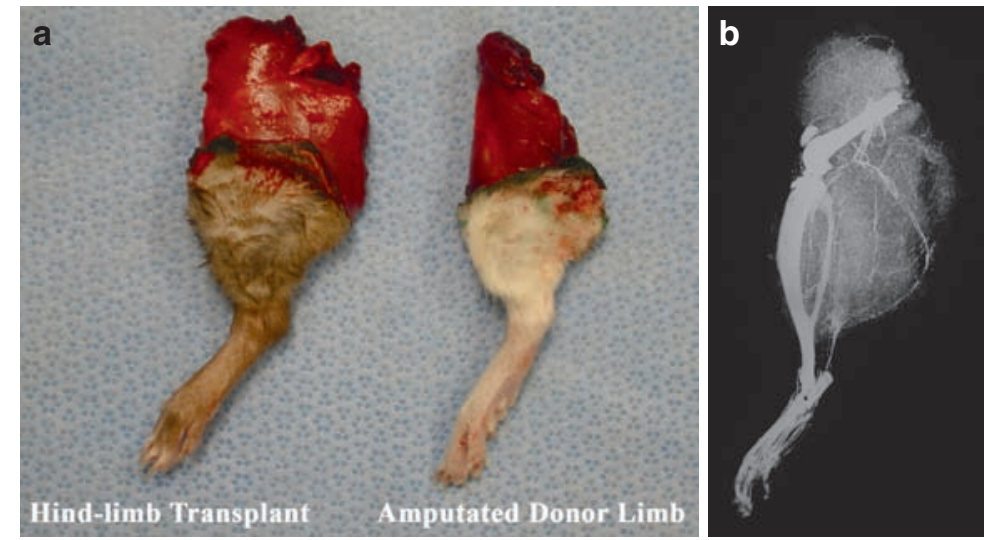

Figure 51.1. (a) Hind-limb model and amputated recipient and is the same size as that of the transplant.

(b) Microangiographic view of hind-limb transplant. (c) Recipient rat after transplant transfer.

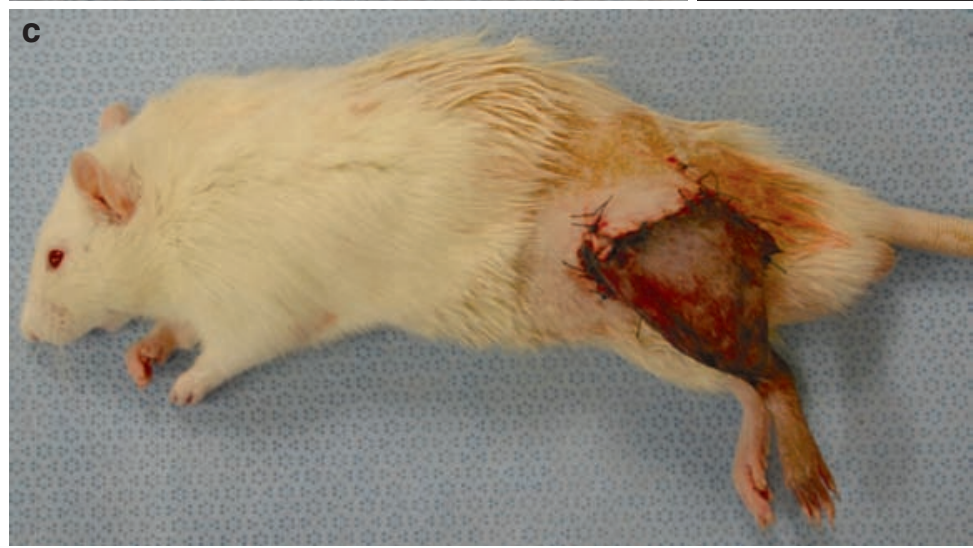


a functional deformity. In this complex model, half of the femur and the entire tibia from the donor are sources for VBMT. Technically, the tibia is transplanted as an intact part of the graft; in contrast, the distal half of the donor femur is rigidly fixed to the proximal half of the recipient's femur. The fixation using a pin and steel wire often damages bone marrow, and as a result the femoral bone is not intact and is not able to adequately contribute to VBMT. ${ }^{4}$ Some investigators have reported major complications such as respiratory failure, bleeding, and thrombus formation. As a result, the published mortality rates range between 20 and $30 \%{ }^{3,8}$

\section{Vascularized Femoral Bone Models}

\section{Vascularized Femoral Bone Model}

Suzuki et al. ${ }^{20}$ described a vascularized femoral bone marrow transplant model, and in this model, the femur was placed in the intraperitoneal space. Tai et al. ${ }^{21}$ developed an extraperitoneal VBMT model that was placed in a subcutaneous pocket over the anterior abdominal wall of the recipient to obviate the surgical difficulties of intraperitoneal placement. We modified Tai et al.'s technique to reduce recipient morbidity, and no subcutaneous pockets were created and no sutures were applied for bone fixation. ${ }^{1}$

Surgical technique: The skin covering the thigh region of the donor limb is degloved. The femur is disarticulated from the knee joint. Anterior, posterior, and lateral thigh muscles are removed and the periosteum is preserved. The adductor muscles are gently dissected to protect the muscular branch of the femoral artery and superior genicular artery, which supplies blood to the femoral bone. Finally, the hip joint is disarticulated, medial and lateral circumflex arteries are ligated, and femoral vessels are prepared for anastomosis. In the recipient, the transplant is transferred to the intra-abdominal space ${ }^{20}$ or groin region, ${ }^{21}$ and abdominal aorta and inferior vena cava or femoral vessels, respectively, are used as recipient vessel (Figure 51.2a).

\section{Bilateral Vascularized Femoral Bone Model}

For augmentation of transplanted bone marrow cells, we have made further modifications by creating bilateral femoral bone allografts based on the common pedicle of the abdominal aorta and inferior vena cava. ${ }^{2}$ In this technique, VBMT must be transferred into the abdominal cavity due to insufficient space for both femoral bones in the groin region of the recipient (Figure 51.2b).

\section{Composite Vascularized Skin/Bone Graft Model}

To augment the antigenic load of a vascularized femoral bone model, we simultaneously harvested the groin skin flap based on the common arterial-venous pedicle. Furthermore, the skin component of the flap facilitated monitoring of the viability of the vascularized bone as well as providing visual indications of rejection. In this model, the vascularized femoral bone is elevated using the standard technique described above while preserving the superficial epigastric artery during groin flap elevation to protect its blood supply (Figure 51.2c).

Advantages of vascularized femoral bone models: Transplantation can be made safely, easily, and without complications. In this transplantation, no nerves, muscles, or bones are transected, and the recipient can use the limb immediately after transplantation without significant morbidity.

Disadvantages of vascularized femoral bone models: Transplant viability cannot be monitored in vascularized femoral bone models without the skin component, because vascularized bone is embedded under the skin and is not able to be visualized for assessments. Bone marrow cell load in this model is lower compared with the limb transplant model (Table 51.3). Vascularized femoral bone transfer does not simulate CTA transplant, because it contains only one tissue component and a lower antigenic load when compared with the more characteristic types of CTA.

\section{Vascularized Iliac Osteomyocutaneous Transplant Model}

To monitor the tolerogenic effect of VBMT, an experimental model should attain both a high antigenic load and be associated with low mortality and morbidity. To achieve this aim, we introduced an iliac osteomyocutaneous VBMT model that contains iliac bone as well as a large abdominal wall muscle component and large skin component.

Surgical technique: In the donor rat, a squareshaped skin island, measuring approximately $8 \times 8 \mathrm{~cm}$, is marked over the flank and lateral 

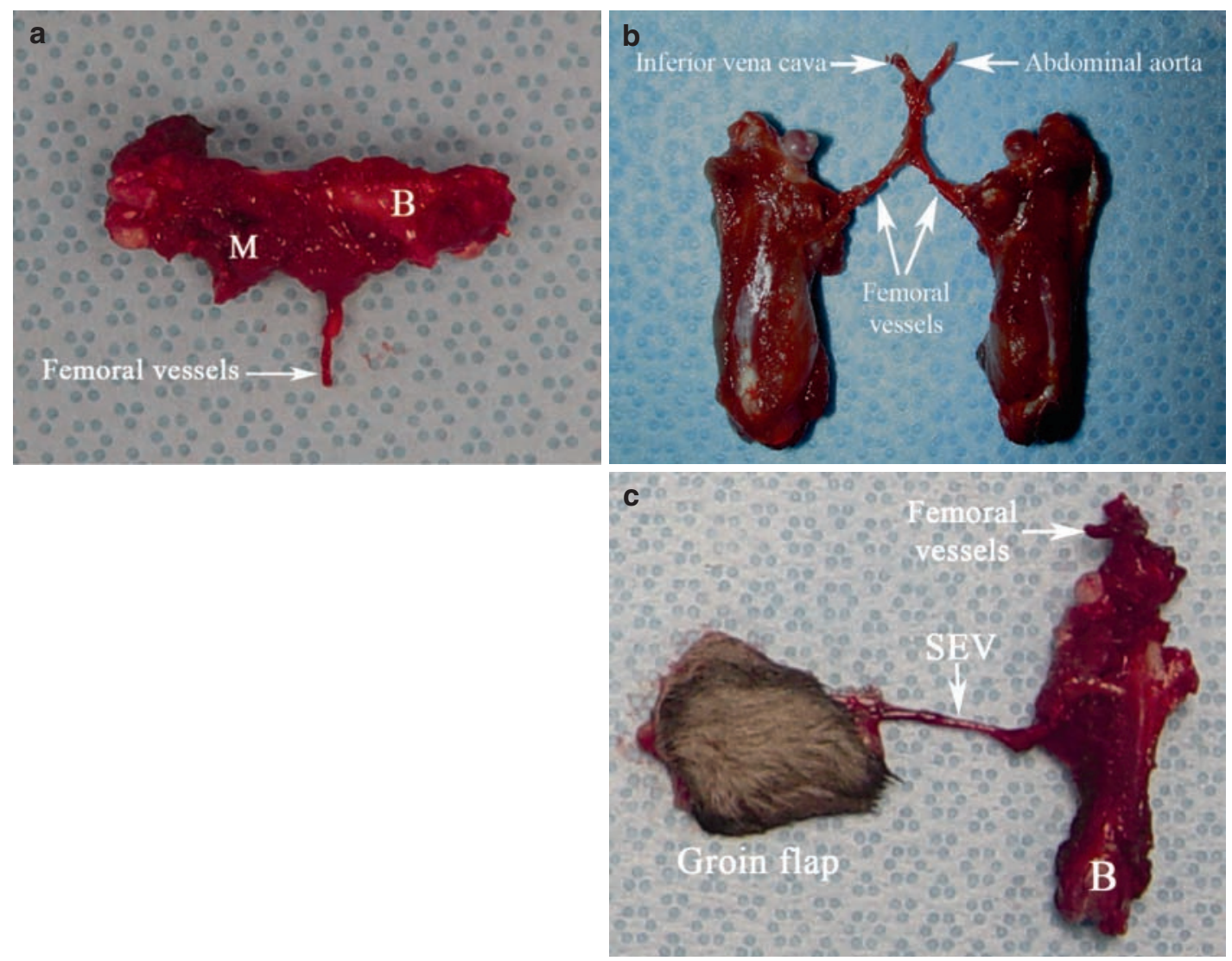

Figure 51.2. Vascularized femoral bone models. (a) Vascularized femoral bone. (b) Bilateral vascularized femoral bone. (c) Composite vascularized skin/bone model. M, muscle component, B, femoral bone. SEV, superficial epigastric vessels.

aspect of the hip of the rat. The skin paddle is raised as an island flap based on the cutaneous perforator of the iliolumbar vessels. Incising the abdominal muscles adjacent to the iliac bone, the vascular pedicle of iliolumbar vessels is exposed and dissected to their origins from the abdominal aorta and the inferior vena cava. Iliac bone osteotomy is performed, and whole iliac bone measuring $3 \times 2 \mathrm{~cm}$ is elevated with the flap. All components of the iliac osteomusculocutaneous flap, including the whole iliac bone, adjacent gluteus maximus, iliacus, lateral abdominal wall muscles, and the overlying skin island, are elevated on the iliolumbar vascular pedicle as a single unit (Figure 51.3).To avoid the smaller caliber iliolumbar artery, the abdominal aorta is used for continuity of pedicle and is transected at the level below the renal artery. However, the iliolumbar vein is used as the pedicle vein, because its diameter is adequate for microvenous anastomosis. In the recipient rat, the groin region is preferred as a recipient area, and the flap pedicle is anastomosed to femoral vessels using end-to-end technique.

Advantages: Iliac bone contains a rich supply of bone marrow cells, and thus it is a viable model for VBMT studies, with a straightforward surgical approach in the recipient and lower rat mortality when compared with limb transplant and sternum models. An iliac osteomusculocutaneous transplant with the largest skin component may present a model of a high antigenic load compared with previously described VBMT models (Figure 51.4) (Table 51.3). Furthermore, it successfully simulates clinical CTA models.

Disadvantages: In our study, we found a lower chimerism level in peripheral blood despite the high bone marrow load found in transplanted bone. In addition, the difference in vessel size 

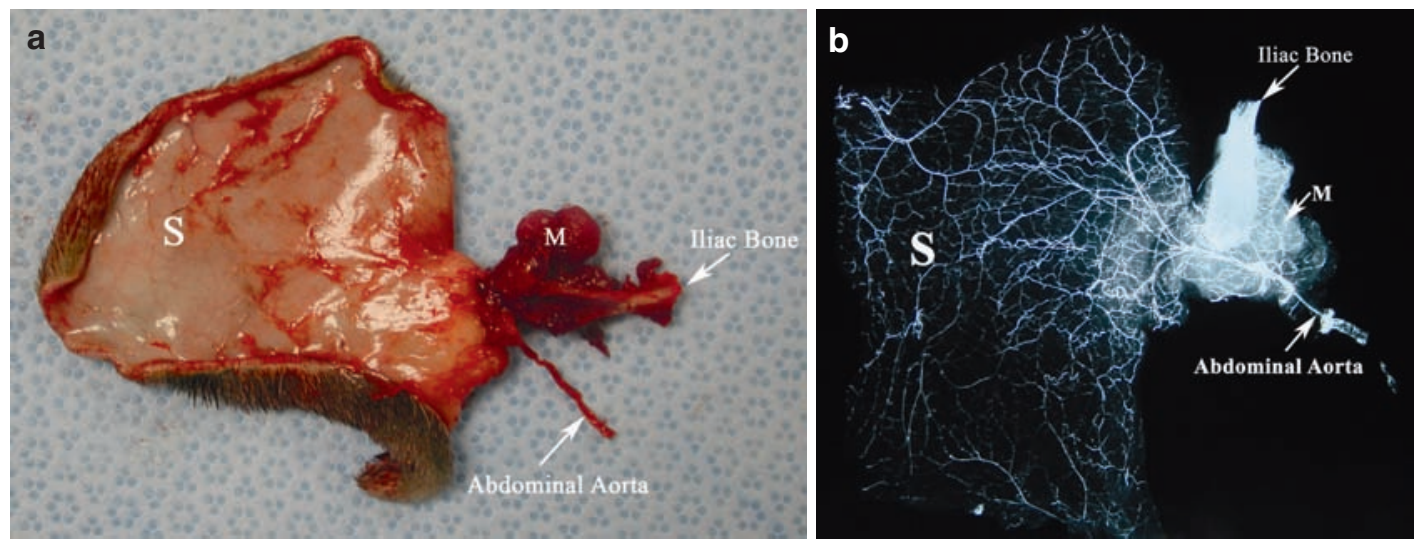

Figure 51.3. (a) Vascularized iliac osteomyocutaneous model. (b) Microangiographic view of transplant components. M, muscle component, S, skin.

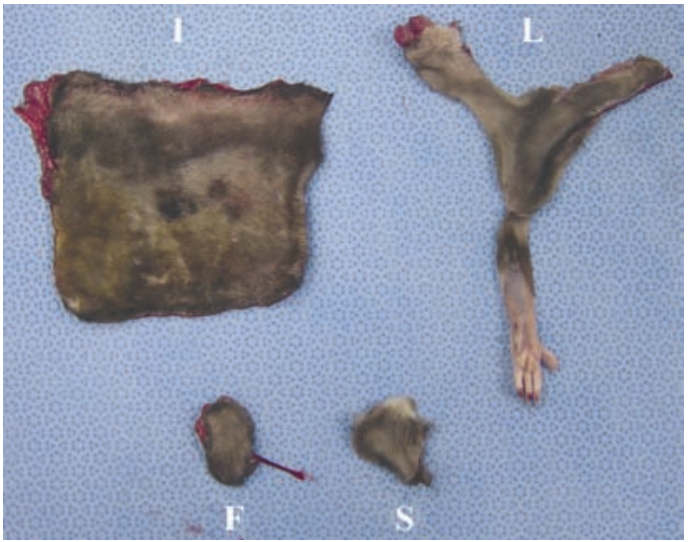

Figure 51.4. Comparison of skin islands of vascularized bone marrow models. Iliac osteomusculocutaneous model has the largest skin island, which is followed by hind limb. I, iliac osteomusculocutaneous, L, limb, $\mathrm{F}$, vascularized femoral bone, $\mathrm{S}$, osteomyocutaneous sternum-thymus model.

Table 51.3. Comparison of VBMT models

\begin{tabular}{lccc}
$\begin{array}{l}\text { VBMT } \\
\text { models }\end{array}$ & $\begin{array}{l}\text { Diameter of } \\
\text { skin island } \\
\left(\mathbf{c m}^{2}\right)\end{array}$ & $\begin{array}{l}\text { Weight } \\
(\mathbf{g})\end{array}$ & $\begin{array}{l}\text { Bone } \\
\text { marrow cell } \\
\text { populations }\left(\mathbf{1 0}^{6}\right)\end{array}$ \\
\hline Iliac & 57.96 & 15.70 & 25 \\
Limb & 24.12 & 18.84 & 48.75 \\
Femur & 5.4 & 14.80 & 50 \\
Sternum & 5.04 & 2.4 & 7.5 \\
\hline
\end{tabular}

between the transplant pedicle and recipient may produce additional challenges during microsurgical anastomosis.

\section{Vascularized Sternum Transplantation Models}

\section{Heterotropic Sternum Transplant Model}

Santiago et al. ${ }^{14}$ described a vascularized sternum transplantation method in which the transplant was transferred into the abdominal cavity as a heterotopic graft.

Surgical technique: In the donor rat, the skin is incised in the midline from the suprasternal space to just above the pubis, and pectoral muscles are divided from the thoracic wall. After entering the peritoneal cavity, the diaphragm is incised, and the thoracic cavity is entered under the manubrium sterni. From the xiphisternum, the ribs on both sides of the sternum are divided $0.5 \mathrm{~cm}$ away from the lateral sternal border up to the clavicle. The entire sternum is then turned cephalad, and the superior vena cava (SVC) is dissected on both sides up to the formation of the brachiocephalic veins. To create a single venous orifice for anastomosis, one SVC is divided distal to the preserved vein and venovenous end-to-side anastomosis is carried out between the left and right SVC. The right and left carotid arteries and subclavian arteries distal to the 
branch of the sternum are ligated and divided. The arteries supplying the sternum are taken with the aortic arch, which is closed distally with a ligature, leaving the proximal end open for the arterial anastomosis. Once the artery and vein are ready, the sternum is separated from the clavicles and soft tissue with a sharp dissection. All raw areas on the graft (cut ends of ribs, manubrium, and divided muscles) are cauterized with caution to control bleeding after transplant transfer (Figure 51.5a). In the recipient, for vessel preparation, the abdomen is opened, followed by isolation of the infrarenal aorta and the inferior vena cava. Sternal transplant pedicle is anastomosed to the recipient vessel the using end-to-end technique.

\section{Vascularized Osteomyocutaneous Sternum-Thymus Model}

To avoid the disadvantages of the heterotopic sternum transplant model and increase antigenic load, we have recently developed a vascularized osteomyocutaneous sternum-thymus model in our laboratory.

Surgical technique: In the donor rat, the skin island and pectoral muscle on the thoracic cage are isolated from the heterotopic sternum transplant, and they are reached through the thoracic cavity by transecting the diaphragm. Brachiocephalic vessels are ligated in the axilla. The ribs on both sides of the sternum are divided from the lateral sternal border up to the clavicle, and the sternum is turned cephalad. A Vessel connection between the thymus and sternum is protected, and the distal ends of the thoracic aorta and inferior vena cava are ligated below the heart. A different method, unique from the previously described model, does not involve dissection of the superior vena cava, and the common carotid artery and external jugular vein are prepared as a transplant pedicle on one side of the neck region. After pedicle preparation, the osteomyocutaneous sternum-thymus composite transplant is divided from the clavicles and soft tissue by sharp dissection (Figure $51.5 \mathrm{~b}, \mathrm{c})$. In the recipient rat, the groin region
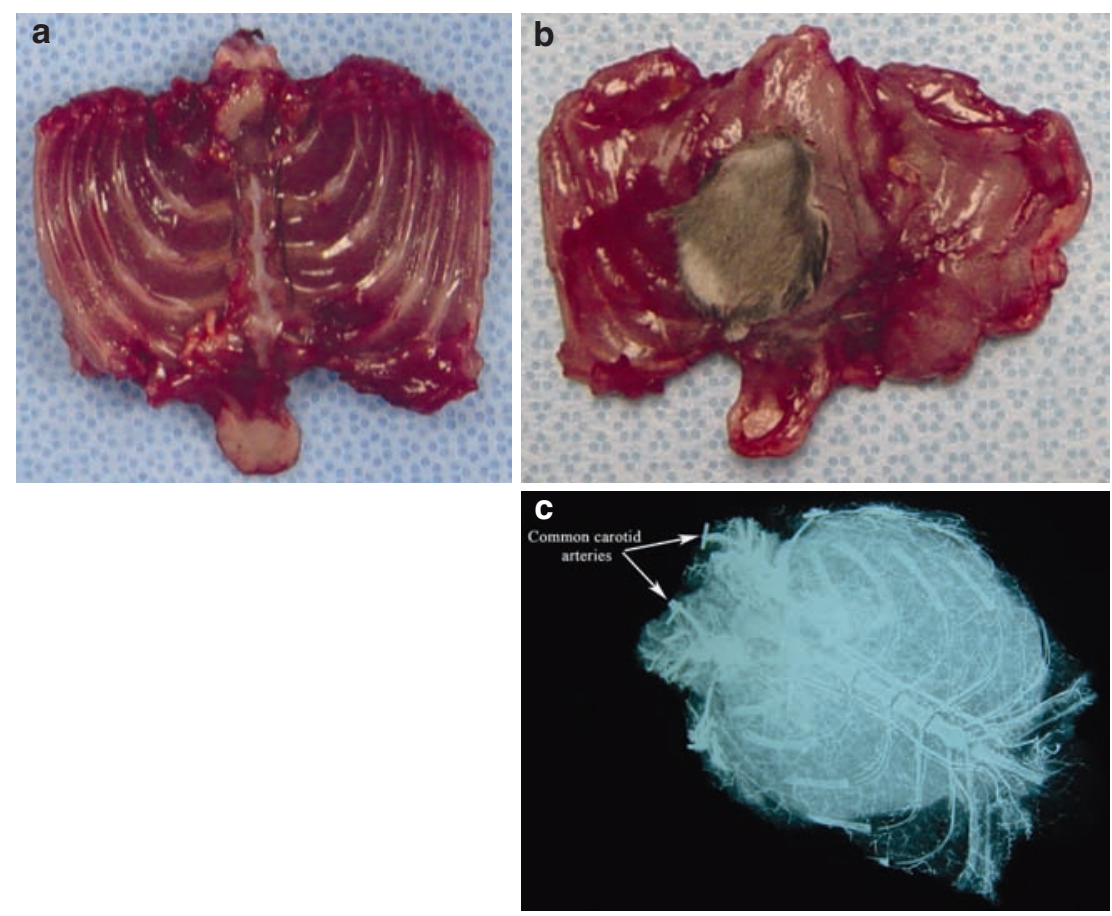

Figure 51.5. Vascularized sternum transplantation models. (a) Vascularized sternum. (b) Vascularized osteomyocutaneous sternum-thymus. (c) Vascular network of vascularized osteomyocutaneous sternum-thymus in the microangiographic view. 
and femoral vessels are prepared for transplant transfer, and an end-to-end technique is used for microanastomosis.

Advantages and disadvantages of vascularized sternum transplantation models: The sternum is a marrow-rich bone and a valuable VBMT model. A salient disadvantage is that the vein used in the vascular pedicle preparation before flap transfer requires an extra microvenous anastomosis between the right and left SVC. Furthermore, pedicle anastomosis with recipient vessels in the abdominal region requires advanced microsurgical skills. Another disadvantage of this model is that assessment of the heterotopic sternum transplant during allotransplant follow-up is impossible due to its intra-abdominal placement. The vascularized osteomyocutaneous sternum-thymus model allows avoidance of a laparotomy and permits externalization of the transplant for convenient observation during the follow-up period, because the transplant is transferred to the groin region. This model presents a high antigenic load due to multiple tissue components, including skin, muscle, and thymus, as well as vascularized bone marrow. Although there is an increased antigenic load in this model, it is still lower when compared with that of the limb transplant model, which has large skin, muscle, and bone components. We found that this model had the lowest bone marrow cell load compared with those in other VBMT models (Figure 51.6) (Table 51.3).

\section{Vascularized Skin Allografts}

Most of the experimental CTA models contain a skin component that induces strong immunologic response from the host. To resolve this immunologic challenge, different vascularized skin allograft transplants are described.

\section{Face Transplantation Models}

Recently, we have described for the first time full-face and hemiface allotransplantation in a rodent model. ${ }^{6,17}$ Subsequent studies included incorporation of different cranial bones (calvarial bone ${ }^{24}$ and mandible ${ }^{9}$ into the facial transplants as a source of VBMT without recipient conditioning.

\section{Full-Face Transplantation Model}

Surgical technique: In the donor rat, first, a median skin incision is made in the neck region. Branches of the external jugular vein between the cranial base and above the clavicle are ligated and the vessel dissected from the subcutaneous tissue. The Sternocleidomastoid muscle is divided and common carotid artery dissected from the vagus nerve. The stylohyoid and omohyoid muscles and the greater horn of the hyoid bone are resected. The full-face transplant is supplied by two main vessels, the facial artery and the posterior auricular

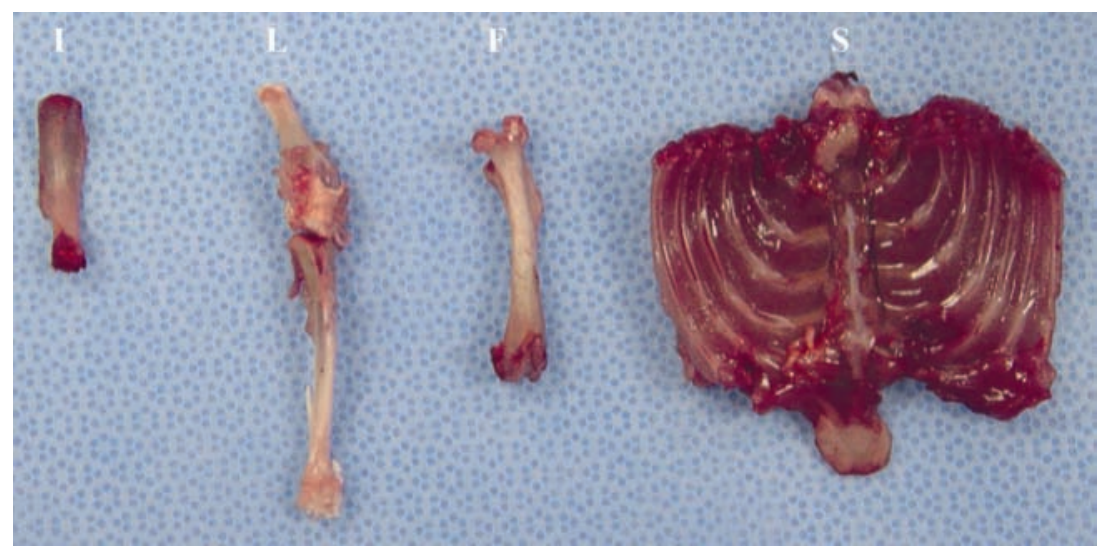

Figure 51.6. Comparison of bone compartment of vascularized bone marrow transplant models. Iliac osteomusculocutaneous and vascularized femoral bone models contain whole bone. Hind-limb transplant models include whole tibia and half of femoral bone, whereas vascularized sternum models covered near-total thoracic cage. I, iliac osteomusculocutaneous, L, limb, F, vascularized femoral bone, S, osteomyocutaneous sternum-thymus model. 
artery, which are branches of the external carotid artery. Dissection of these vessels is the most important part of the face harvesting procedure. After ligation of the internal carotid artery and the cervical branches of the external carotid artery in the neck region, the facial artery is dissected up to the point of its emergence from the external carotid artery. This dissection is continued to the nasal region, which is called the angular artery at this level. After circular neck incision, to facilitate dissection of posterior auricular artery, the ear is detached at the level of the external cartilaginous auricular canal, and this vessel is elevated with skin from the ear. At the same level, the branches of the posterior facial vein, which continue as the external jugular vein, are divided from the pterygoid and pharyngeal vein plexuses. Then the facial allograft transplant, which includes ear, neck, and facial skin without eyelid and nose skin, is completely freed from all surrounding tissues based on the bilateral common carotid artery and external jugular vein. The dissections of the branches of the facial or trigeminal nerve are optimal. In the recipient, the same size of facial skin is excised. Bilateral common carotid arteries and external jugular veins of the full-face transplant pedicle are anastomosed with the recipient bilateral common carotid arteries and external jugular vein respectively, using the end-to side technique (Figure 51.7a, b, c). ${ }^{17}$
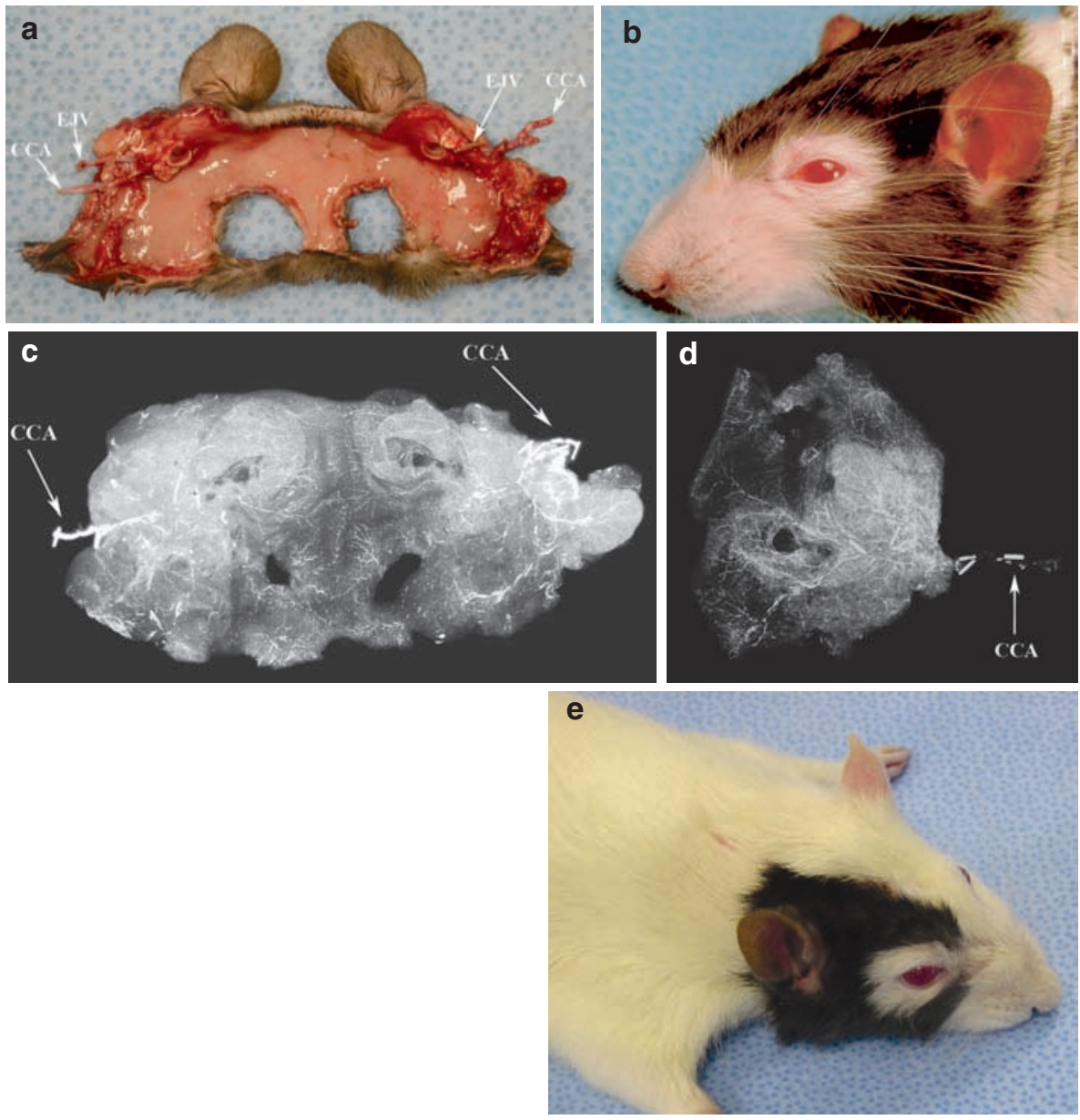

Figure 51.7. Face transplantation models. (a) Harvested full face (b) Transplanted full-face model. (c, d) Microangiographic views of full- and hemiface transplants. (e) Transplanted hemiface.CCA, common carotid artery; EJV, external jugular vein. 


\section{Hemiface Face Transplantation Model ${ }^{6}$}

Surgical technique: All surgical procedures are the same as those in the full-face transplantation. However, the transplant contains only the hemiface skin, and, therefore, preparation of a unilateral common carotid artery and external jugular vein is sufficient as a transplant pedicle. Transplant arteries are anastomosed to the recipient common carotid using the end-toside technique, whereas the vein anastomosis is performed using an end-to-end technique (Figure $51.7 \mathrm{c}, \mathrm{d}$ ). ${ }^{6}$

Advantages and disadvantages of facial transplantation models: Full-face allograft transplantation in the rat model has higher complication and mortality rates as a result of brain ischemia and vascular complications. In this technique, the main vascular structures of the head and neck are used bilaterally on carotid arteries and jugular veins in the recipient. To avoid this hazardous disadvantage and for the purpose of reducing mortality in facial allograft recipients, we have introduced another modification to the face allotransplantation model, in which a total facial allograft flap was based on bilateral common carotid arteries and was vascularized based on the unilateral common carotid artery of the recipient. ${ }^{22}$ In the hemiface models, arterial circulation of the brain was not significantly affected, because only one major vessel of the head and neck was used, and the flap was connected to the recipient's pedicle via the end-to-side technique. This model may be more suitable for immunologic studies instead of the full-face model, because it contains the same tissue components as a full-face model without the associated morbidity and mortality found in the full-face model.

\section{Combined Hemiface-Bone Transplantation Models}

In order for our experimental face transplantation studies to achieve longer survival time, facial bone components were added to the hemiface models. Furthermore, we achieved face and vascularized bone transplantation at the same time, and thus we intended to examine the benefits of heterotopic bone marrow on immune tolerogenicity.

\section{Composite Hemiface/Calvaria Transplantation Model}

Surgical technique: In this model, the parietal bone of a rat is added to the hemiface transplant. The hemiface is elevated using the technique described previously. However, connections between the periosteum of the parietal bone and transplant tissues are protected, and the bone is harvested combined with a hemiface transplant. The composite flap is transferred using the common carotid artery and external jugular vein with the same microsurgical technique used in the hemiface transplant (Figure 51.8a, b). ${ }^{24}$

\section{Composite Hemiface, Mandible, Tongue Tissue Allograft Model}

The flap harvesting technique is the same as that in the hemiface model, and the same vessels are used to transplant the pedicle. A unique step that differs from the previous technique is whereby the hemiface is elevated in combination with both the hemimandible and tongue tissue. During transplant elevation, connections between the transplant and hemi mandible and tongue are maintained while preserving the blood supply, and these parts are harvested in combination with the hemiface transplant. The groin region and femoral vessels are prepared as recipient, and the transplant is transferred using the end-to-end technique (Figure 51.8c, d). ${ }^{9}$

Advantages and disadvantages of combined hemiface-bone transplantation models: Both methods are used successfully for bone marrowbased therapy studies, as demonstrated by prolonged survival time and increased chimerism levels. Furthermore, the mandibular segment of the composite hemiface, mandible, tongue tissue allograft model is suitable for prospective orthognathic surgery. However, creation of the same defect in a recipient rat in this model would make survival impossible, and thus there is no chance for orthotopic transplantation.

\section{Vascularized Groin Flap}

A groin flap (or inferior epigastric flap) is the first described experimental free flap model. ${ }^{18}$ Although it is accepted as a vascularized skin 

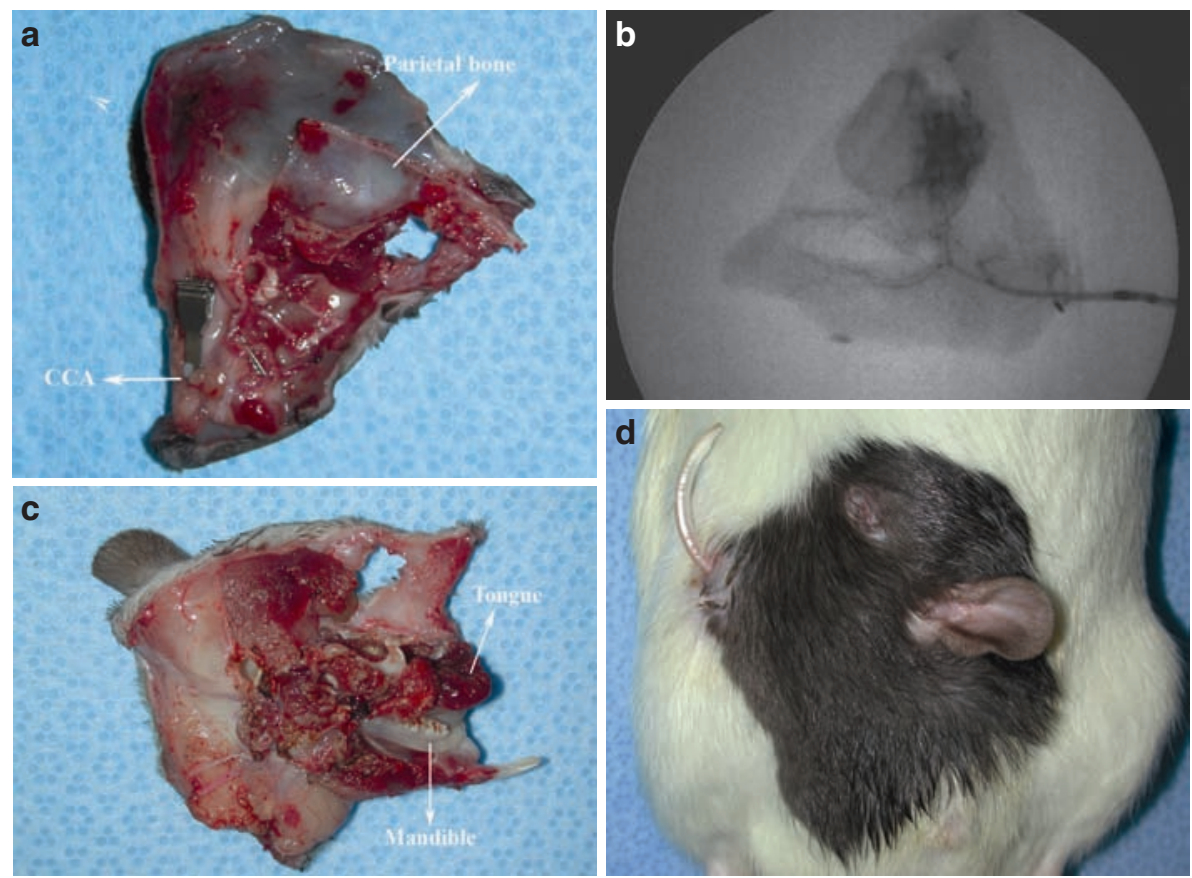

Figure 51.8. (a) Composite hemiface/calvaria. (b) Microangiographic view of composite hemiface/calvaria transplant. (c) Composite hemiface, mandible, tongue tissue. (d) Transplanted composite hemiface, mandible, tongue model. CCA. common carotid artery.

allograft, the inguinal fat pad constitutes an important part of flap volume.

Surgical technique: In the donor rat, the groin and lower abdominal skin are used as the vascularized allograft design. After the skin incision, a skin island with inguinal fat pat is elevated on the anterior abdominal wall muscle. The groin transplant pedicle has a superficial inferior vessel, which is dissected at its origin on the femoral vessels. Due to the large vessel diameter and a pedicle length, femoral vessels are ligated from the distal region of the superficial epigastric vessels' origins and divided during transplant elevation (Figure 51.9).

Advantages: This model is very useful for immunotolerance studies because of its simple surgical dissection, low rat morbidity, and mortality.

Disadvantages: When the skin island section is extended on the contralateral side and passes the abdominal midline, partial necrosis is observed, because its pedicle provides blood supply only to the same side lower abdominal and groin skin. Thus, maintaining a large skin island section is impossible using this flap.

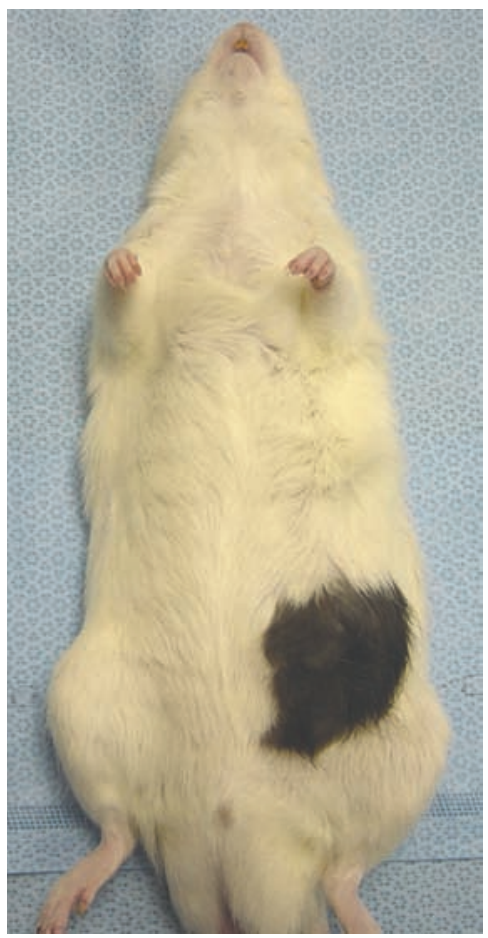

Figure 51.9. Transplanted groin flap in recipient rat. 


\section{Extended Groin Flap Model}

Although definitive data has not yet been published, it is thought that larger amounts of skin tissue in CTA transplants may increase the antigenic load. An experimental model that has a large skin component may be used to test this hypothesis. We have described such a model using an extended groin flap to provide a large skin island for transplantation study.

Surgical technique: The Skin island is planned measuring approximately over the abdominal wall and flank regions in the donor rat. The borders of the skin island are defined superiorly by the costal cage, inferiorly by the inguinal ligament, and $3 \mathrm{~cm}$ below the iliac spine in flank regions, respectively, medially by abdominal midline, and laterally by dorsal midline. After skin incision, elevation starts at the abdominal section on the anterior abdominal wall, and dissection is continued with flank section elevation over the lateral abdominal wall muscles. Iliolumbar vessels are found close to the upper border of the iliac bone and are cauterized and separated so that their connection with the superficial epigastric artery maintains blood supply to the flank region. After this step, the flap harvesting procedure is the same as that with the previously described groin flap (Figure 51.10).
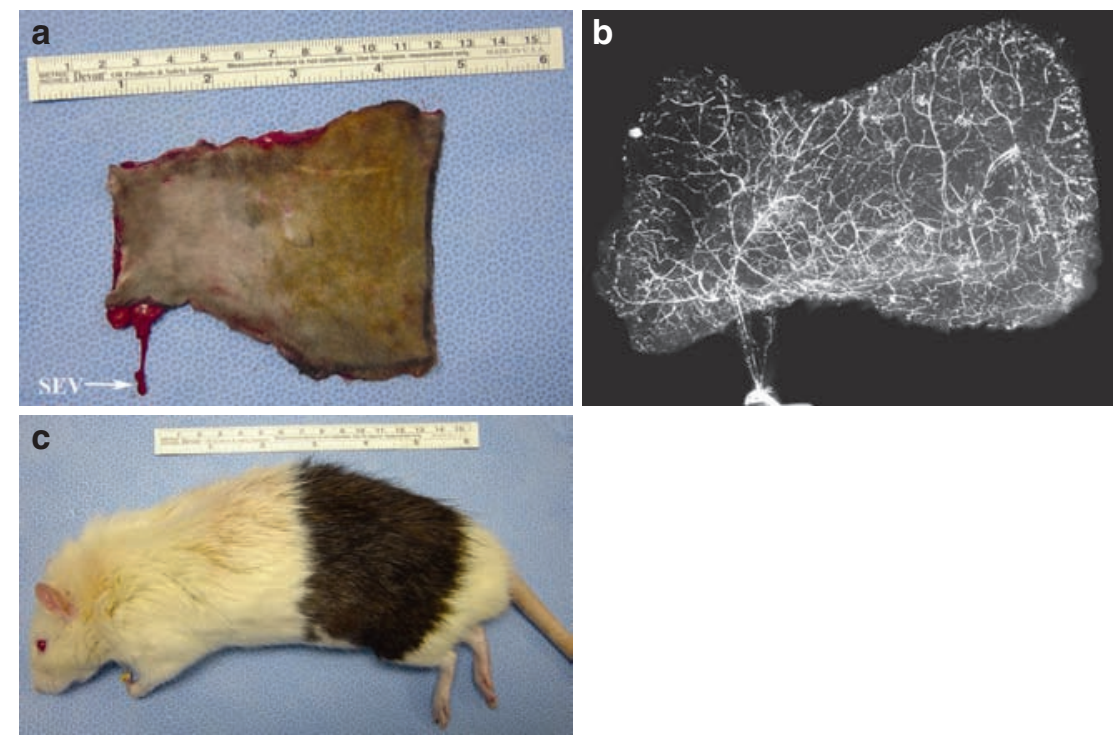

Advantages: This model has a short operation time and minimal rat mortality. Although a large amount of skin is used, the design of this skin flap makes it possible to harvest two flaps from the same animal and thus reduces the number of necessary donors.

Disadvantage: Seromas may collect under transplant tissue because of the large dead space that underlies it. To avoid seroma complications, a few sutures may be placed between the flap and basement tissue to decrease the amount of dead space.

\section{Total Abdominal Wall Transplant Model}

In all experimental models in the rat, the total abdominal wall transplant model entails the largest skin component. We described this model to evaluate immunologic response against a large skin size. ${ }^{12}$

Surgical technique: In the donor rat, the skin island of this model covers the entire abdominal skin region. The border of the skin island extended from the xiphoid process and arcus costa to the pubic tubercle and inguinal ligaments and between the two anterior axillary lines. Following incision of the skin island, the total abdominal transplant was elevated over the anterior abdominal

Figure 51.10. (a) Extended groin flap includes wide skin island, which covers groin, lower abdomen, and flank regions. (b) Microangiographic view of extended groin flap. (c) Recipient rat after transplantation after long term follow-up. 
wall muscle. Pedicle dissection is the same as that with the groin flap, but bilateral superficial epigastric vessels are prepared as the flap pedicle. In the recipient rat, all of the anterior abdominal skin is harvested, and the bilateral femoral vessels are dissected for flap transfer. Bilateral flap pedicle is anastomosed to the femoral vessels on both sides using the end-toend technique. A few fixation sutures are placed between the transplant and abdominal muscle in the midline for prevention of seroma collection (Figure 51.11).

Advantages: The surgical procedure is very similar to that in the previously described groin flap procedure, with the exception that there is a need for bilateral microanastomosis. Furthermore, the surgery required in the preparation of the large vascularized skin transplant is relatively simple. This is the first model demonstrating that an abdominal wall transplant could be used to evaluate causes of high rejection rates in clinical transplants.

Disadvantages: This model led to a higher morbidity and mortality rate compared with the groin and extended groin transplants. The large recipient skin excision needed to create the defect for transplant transfer and large tissue transfer may have resulted in severe hemodynamic imbalance in the recipient.

\section{Muscle Transplantation Models \\ Cremaster Muscle CTA Transplantation Model}

This CTA transplantation model was developed to study the microcirculatory changes during acute allograft rejection and ischemia/reperfusion
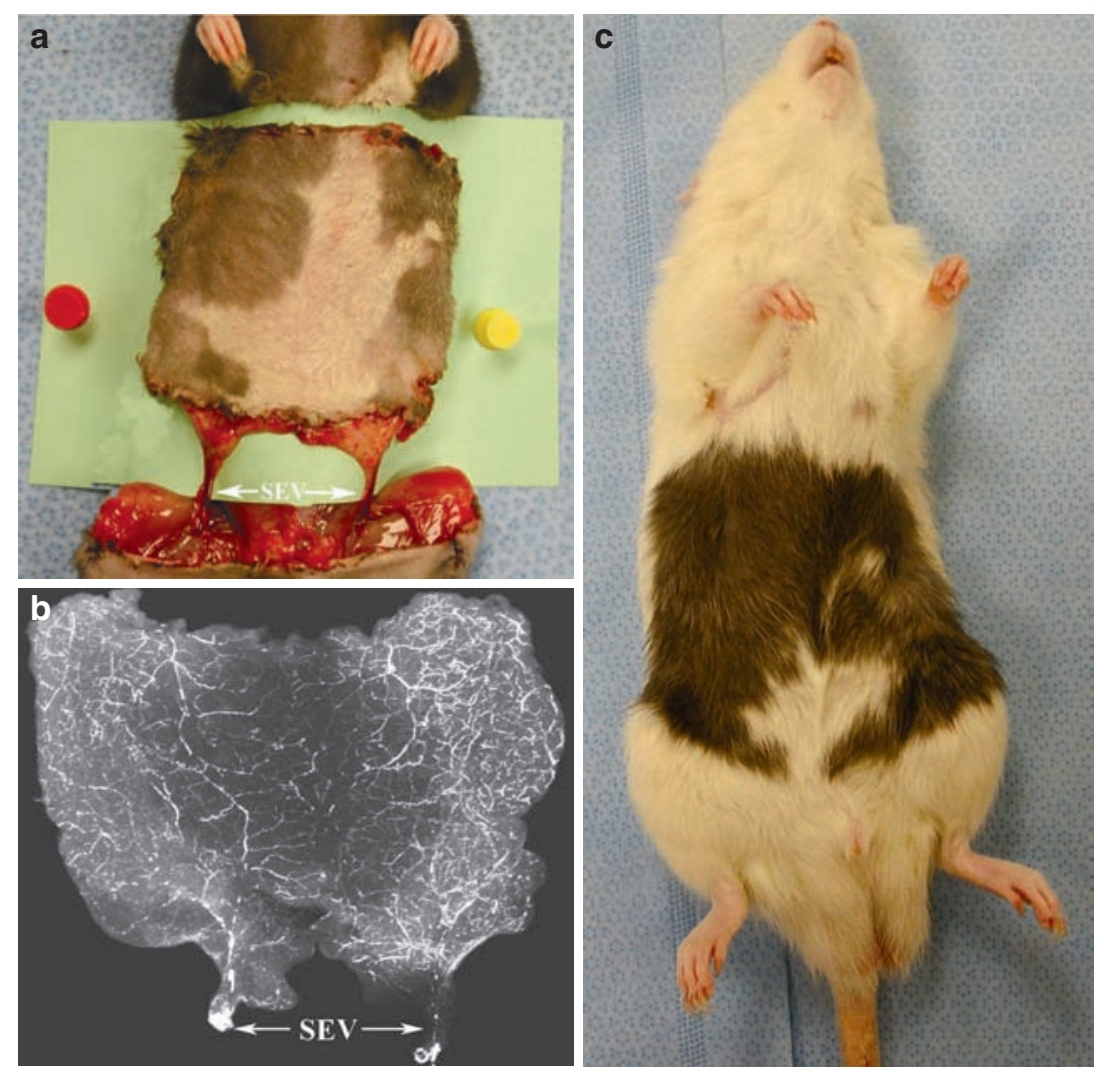

Figure 51.11. (a) Total abdominal wall transplant model based on bilateral superficial epigastric pedicles. Entire abdominal skin of recipient is elevated for total abdominal wall transplant. (b) Wide microvascular network of vascularized transplant model. (c) Recipient rat after transplantation after long-term follow up. SEV, superficial epigastric vessels. 
injury in our laboratory. ${ }^{13}$ This model is the first described one performed on the mouse cremaster transplant model.

Surgical technique: An anterior longitudinal skin incision is made starting from the testicle to the xiphoid in the donor rat. After identification of the cremaster muscle on its vascular pedicle, the testicular vessels are ligated and the testicular contents are extracted. The cremaster muscle is isolated as a tube flap. The pudic-epigastric branch of the femoral artery, the main feeding branch of the flap, is identified, and the side branches are ligated. The distal branch of the femoral artery and the vein are tied, and the cremaster muscle is elevated on the common iliac artery and vein. The cremaster muscle CTA model is transplanted to the neck region of the recipient, and the pedicle is anastomosed to the recipient's ipsilateral carotid artery and external jugular vein using the end-to-end technique (Figure 51.12).

Advantages: Mouse studies of pathophysiologic states have some advantages over those with other animals. Housing, maintenance, and treatment costs of mice are much less, compared with other laboratory animals. Treatment modalities applied per body weight of the animal are 5-10 times less expensive in mice than those in rats.

Disadvantages: The vessel size of the donor and recipient arteries is very small compared with that in rat, and this surgery needs advanced microsurgical skill. Mice are less tolerant to surgical trauma, blood loss, and anesthesia and have a higher mortality rate than rats. Meticulous attention should be paid to minimize blood loss during surgery.

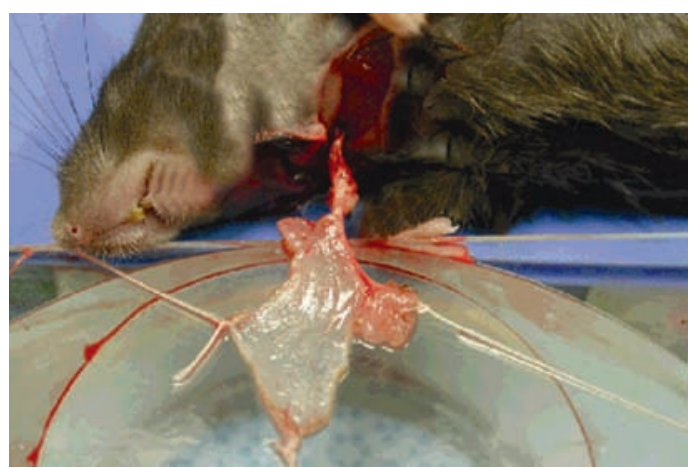

Figure 51.12. Transplanted cremaster muscle flap in the neck region of the muscle.

\section{Other CTA Models}

\section{Maxilla Allotransplantation Model}

Face bone transplantation may serve as an alternative option in clinical transplantation in the future. With this in mind, we have described a maxilla allotransplantation model to evaluate the immunologic responses and growth properties of transplanted face bones. ${ }^{23}$

Surgical technique: In the donor rat, after skin incision, the external carotid artery and its branches are dissected by the midline neck approach.After isolating the common carotid artery and the bifurcation, the external carotid arteries are ligated and divided bilaterally. External jugular veins are dissected and the side branches ligated from the supraclavicular region to under the cranial base. Finally, the maxilla is dissected along Le-Fort II osteotomy lines based on the common carotid artery and external jugular vein and transplanted to the anterior abdominal wall using end-to-end anastomosis with femoral vessels in the recipient rat (Figure 51.13).

Advantages: Using this model may allow for evaluation of the growth properties of teeth, facial bone, and cartilage in allografts and could provide valuable information with potential clinical applications.

Disadvantage: Orthotopic transplantation for this model is impossible due to the high mortality related to animal suffocation and nutritional problems.

\section{Vascularized Laryngeal Allograft Transplantation Model}

In 1992, Strome et al. ${ }^{19}$ developed a vascularized laryngeal transplantation model to reexamine the potential for laryngeal transplantation.

Surgical technique: For transplant harvesting from donor rat, the skin incision extends from the hyoid bone to the sternal notch. The salivary glands, sternohyoid, and thyrohyoid muscles are excised, and the omohyoid muscle is divided to expose the internal carotid arteries. The external carotid arteries are ligated and severed above the superior thyroid arteries, with the common and internal carotid arteries left intact. Internal carotid artery and ascending pharyngeal arteries are ligated a few millimeters superior to the carotid 

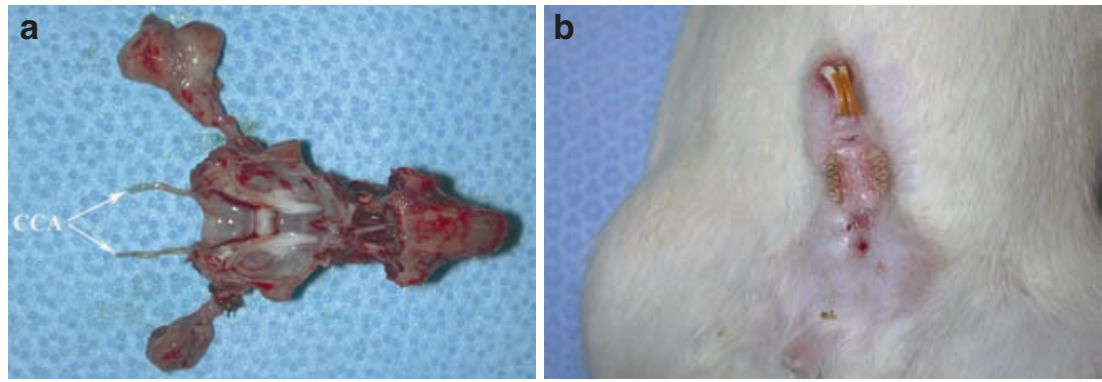

Figure 51.13. (a) Maxilla allotransplantation model. (b) Transplanted maxilla allotransplantation in the groin region.

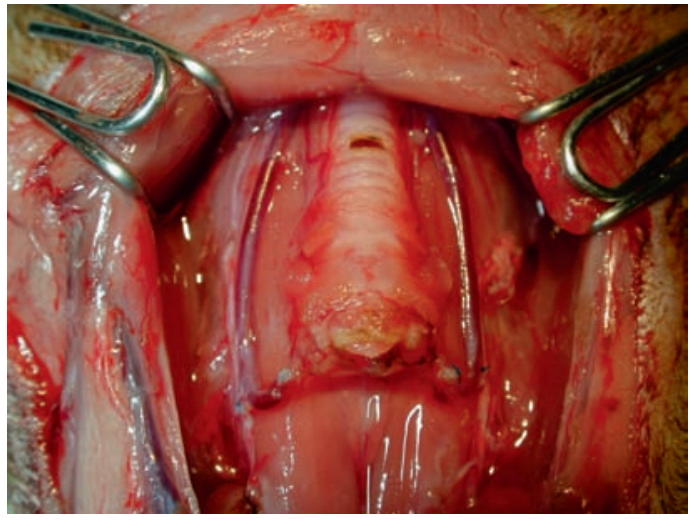

Figure 51.14. Laryngeal allograft transplantation model.

bifurcation. This procedure is performed on the other side, and the lower border of the larynx is freed by transection of the trachea at the second tracheal ring. Then the larynx is dissected from the esophagus from cephalad to the superior thyroid artery until the larynx is connected to the donor rat only by its vascular pedicle. The larynx is removed following bilateral common carotid artery transection. The recipient neck region and vessel techniques are performed as described for the donor rat, with the dissection being limited to the left side. The right common carotid artery is anastomosed to the left common carotid artery using the end-to-side technique. Next, the left common carotid artery of the donor is anastomosed end-to-side to the external jugular vein of the recipient (Figure 51.14).

Advantages: This model is suitable for large trials to determine the fundamental criteria for graft life.

Disadvantage: This procedure needs advanced microsurgical skill and has high postoperative morbidity.

\section{Conclusion}

To open a new category for CTA transplantation in the reconstructive ladder in plastic surgery practice, the experimental CTA model is a very important tool for transplantation studies.

Different models have allowed investigators to evaluate the immunogenicity and possible clinical implications of CTA transplantation using different techniques. Continued research in the development of these and other models will further delineate the role of CTA transplants in plastic and reconstructive surgery.

\section{References}

1. Agaoglu G, Unal S, Siemionow M. Transplantation of the vascularized bone allograft into the inguinal region. Plast Reconstr Surg. 2005;115:1794-1795.

2. Agaoglu G, Carnevale KA, Zins JE, et al. Bilateral vascularized femoral bone transplant: a new model of vascularized bone marrow transplantation in rats, part I. Ann Plast Surg. 2006;56:658-664

3. Arai K, Hotokebuchi T, Miyahara H, et al. Limb allografts in rats immunosuppressed with FK506. I. Reversal of rejection and indefinite survival. Transplantation. 1989;48:782-786.

4. Arslan E, Klimczak A, Siemionow M. Chimerism induction in vascularized bone marrow transplants augmented with bone marrow cells. Microsurgery. 2007;27:190-199.

5. Black KS, Hewitt CW, Woodard TL, et al. Efforts to enhance survival of limb allografts by prior administration of whole blood in rats using a new survival endpoint. J Microsurg. 1982;3:162-167.

6. Demir Y, Ozmen S, Klimczak A, et al. Tolerance induction in composite facial allograft transplantation in the rat model. Plast Reconstr Surg. 2004;114:1790-1801.

7. Hart DN, Fabre JW. Major histocompatibility complex antigens in rat kidney, ureter, and bladder. Localization with monoclonal antibodies and demonstration of Ia-positive dendritic cells. Transplantation. 1981;31:318-325.

8. Hovius SE, van Adrichem LN, van der Heijden, et al. Postoperative monitoring of allogeneic limb transplantation in rats. Ann Plast Surg. 1988;21:559-565. 
9. Kulahci Y, Klimczak A, Siemionow M. Developement of the alternative composite tissue allograft transplantationmodels foe reconstruction of the head and neck defects., 51st Annual Meeting of the Plastic Surgery Research Council, Dana Point, California; 2006.

10. Lee WP, Yaremchuk MJ, Pan, et al. Relative antigenicity of components of a vascularized limb allograft. Plast Reconstr Surg. 1991;87:401-411.

11. Mathes DW, Randolph MA, Solari MG, et al. Split tolerance to a composite tissue allograft in a swine model. Transplantation. 2003;75:25-31.

12. Nasir S, Bozkurt M, Klimczak A, et al. Large antigenic skin load in total abdominal wall transplants permits chimerism induction. Ann Plast Surg. 2008;61(5):572-579.

13. Ozer K, Zielinski M, Siemionow M. New composite tissue allograft transplantation model in mouse with intravital microscopic evaluation of microcirculation. J Reconstr Microsurg. 2003;19:323-330.

14. Santiago SF, de Faria W, Khan TF, et al. Heterotopic sternum transplant in rats: a new model of a vascularized bone marrow transplantation. Microsurgery. 1999;19: 330-334.

15. Siemionow M, Oke R, Ozer K, et al. Induction of donorspecific tolerance in rat hind-limb allografts under antilymphocyte serum and cyclosporine A protocol. $J$ Hand Surg $[\mathrm{Am}] .2002 ; 27: 1095-1103$.

16. Siemionow M, Ortak T, Izycki D, et al. Induction of tolerance in composite-tissue allografts. Transplantation. 2002;74:1211-1217.
17. Siemionow M, Gozel-Ulusal B, Engin Ulusal A, et al. Functional tolerance following face transplantation in the rat. Transplantation. 2003;75:1607-1609.

18. Strauch B, Murray DE. Transfer of composite graft with immediate suture anastomosis of its vascular pedicle measuring less than $1 \mathrm{~mm}$ in external diameter using microsurgical techniques. Plast Reconstr Surg. 1967;40: 325-329.

19. Strome S, Sloman-Moll E, Samonte BR, et al. Rat model for a vascularized laryngeal allograft. Ann Otol Rhinol Laryngol. 1992;101:950-953.

20. Suzuki H, Patel N, Matthews, et al. Vascularized bone marrow transplantation: a new surgical approach using isolated femoral bone/bone marrow. J Surg Res. 2000;89: 176-183.

21. Tai CY, France MA, Strande LF, et al. An extraperitoneal isolated vascularized bone marrow transplant model in the rat. Transplantation. 2003;75:1591-1593.

22. Unal S, Agaoglu G, Zins J, et al. New surgical approach in facial transplantation extends survival of allograft recipients. Ann Plast Surg. 2005;55:297-303.

23. Yazici I, Unal S, Siemionow M. Composite hemiface/ calvaria transplantation model in rats. Plast Reconstr Surg. 2006;118:1321-1327.

24. Yazici I, Carnevale K, Klimczak A, et al. A new rat model of maxilla allotransplantation. Ann Plast Surg. 2007;58: 338-344.

25. Zhang F, Shi DY, Kryger Z, et al. Development of a mouse limb transplantation model. Microsurgery. 1999;19:209-213. 